\title{
Recommendations for Diagnosis and Management of Osteoporosis in COPD Men
}

\author{
Elias E. Mazokopakis ${ }^{1}$ and Ioannis K. Starakis ${ }^{2}$ \\ ${ }^{1}$ Department of Internal Medicine, Naval Hospital of Crete, Chania, 73200 Crete, Greece \\ ${ }^{2}$ Department of Internal Medicine, Patras University Hospital, 26500 Rion-Patras, Greece
}

Correspondence should be addressed to Elias E. Mazokopakis, emazokopakis@yahoo.gr

Received 28 April 2011; Accepted 21 June 2011

Academic Editor: L. Gennari

Copyright (C) 2011 E. E. Mazokopakis and I. K. Starakis. This is an open access article distributed under the Creative Commons Attribution License, which permits unrestricted use, distribution, and reproduction in any medium, provided the original work is properly cited.

\begin{abstract}
Patients with chronic obstructive pulmonary disease (COPD) are at increased risk for osteoporosis and fractures because of lifestyle factors, systemic effects of the disease, side effects of treatment, and comorbidities. The initial evaluation of COPD men for osteoporosis must include a detailed medical history and physical examination, assessment of COPD severity, and additional tests, as suggested by results of clinical evaluation. Osteoporosis is diagnosed on the basis of bone mineral density (BMD) measurement with DEXA of the lumbar spine and hip, but fracture risk assessments tools, as FRAX, could be used as useful supplements to BMD assessments for therapeutics interventions. The prevention and treatment of osteoporosis in COPD involves nonpharmacologic and pharmacologic measures, as lifestyle measures and nutritional recommendations, management of COPD treatment (based on the use of limited corticosteroids doses), and drug therapy (bisphosphonates, teriparatide). In this paper, the current recommendations for diagnosis and management of osteoporosis in COPD men, based on recent medical bibliography, are presented and discussed.
\end{abstract}

\section{Introduction}

Chronic obstructive pulmonary disease (COPD) is a major cause of morbidity and mortality worldwide and a secondary cause of bone loss [1-3]. It is characterised by a progressive airflow limitation that is not fully reversible and develops as a consequence of genetic susceptibility, increasing life span, environmental, and lifestyle factors $[1,3,4]$. The degree of airflow limitation can be assessed by spirometry and stratified in accordance with the Global Initiative for Chronic Obstructive Pulmonary Disease (GOLD) [4]. Osteoporosis is a common systemic skeletal disease characterised by low bone mass with microarchitectural disruption and skeletal fragility, resulting in an increased risk of fracture, most commonly at the spine, hip, or wrist and causing significant morbidity and mortality $[3,5]$. Osteoporosis in men continues to be underdiagnosed and untreated due to the rather exclusive focus on postmenopausal osteoporosis in the past; corticosteroid use, excessive alcohol use, and hypogonadism which are the most frequent secondary causes of male osteoporosis [2, 6]. Although osteoporosis is less prevalent in men, it has been estimated that $30 \%$ of all hip fractures occur in males and that one in eight men older than 50 years will experience an osteoporotic fracture [5]. Moreover, many studies have shown that osteoporotic fractures are associated with greater morbidity and mortality in men compared with women $[5,7]$.

The majority of COPD patients in clinical practice are men of older age with many underdiagnosed additional risk factors for osteoporosis. The purpose of this paper is to review the current recommendations for diagnosis and management of osteoporosis in COPD men.

\section{Pathogenesis and Prevalence of Osteoporosis in COPD}

Patients with COPD are at increased risk for osteoporosis and fractures because of lifestyle factors, systemic effects of the disease, side effects of treatment, and comorbidities $[1,8]$. It is marked that COPD and osteoporosis share some common 
risk factors [8]. The current or past tobacco smoking, the inactivity, and weight loss (known as pulmonary cachexia) in COPD patients are associated with decreased bone mineral density (BMD) and increased risk of fractures [1]. Also, COPD itself is an independent predictor of BMD reduction and vertebral fracture risk, and increasing disease severity increases the risk for osteoporosis and fracture risk $[1,3,9]$. It could be explained, as a result of mediators, inflammatory cytokines (interleukin [Il]-6, TNF- $\alpha$ ) which induce expression of RANKL (receptor activator of nuclear factor$\kappa \mathrm{B}$ ligand) and RANKL-mediated bone resorption [10]. In addition, many other cytokines have been found to interact with the OPG (osteoprotegerin)/RANKL system, supporting the concept that inflammatory mediators contribute to the regulation of bone remodelling [10]. Moreover, COPD patients, as well as patients with other chronic diseases, often have deficient calcium $(\mathrm{Ca})$ and vitamin $\mathrm{D}$ nutritional status, and the latter is frequently accentuated because of little exposure to sunlight $[1,10]$. Among available treatments for COPD, only corticosteroid- (CS-) treatment has documented adverse effects of clinical significance $[1,10]$. Oral glucocorticosteroids (OGCS) have both direct adverse effects on bone and indirect effects due to muscle weakening and atrophy. These effects are dose dependent both regarding daily dose, duration, and cumulative dose. Less negative effects are seen by sporadic compared to continuous use, but lower continuous doses have less detrimental effects on bone than frequent high-dose course of OGCS [1]. Systemic OGCS can cause a rapid bone loss within the first few months of treatment, followed by a slower $2-5 \%$ loss per year with chronic use [11]. However, OGCS are recommended only for 7 to 10 days with 30 to $40 \mathrm{mg}$ prednisolone in exacerbations in patients with severe COPD; long-term treatment with OGCS is not recommended in COPD [12]. High doses of inhaled corticosteroids (ICS) seem to increase bone loss, but the extent of this effect is discussed because it is difficult to differentiate between the effect of ICS dose used, other risk factors, prior or concomitant use of OGCS, and not at least, disease severity [1]. A recent study, addressing static and dynamic indices of cancellous and cortical bone structure in postmenopausal women with COPD who had not received chronic OGCS, revealed skeletal microstructural abnormalities in cancellous and cortical bone providing an explanation for the high prevalence of vertebral fractures in this disease [13]. It is important to prevent fractures in COPD patients, considering that hip and vertebral fractures might impair mobility, and vertebral fractures further reduce lung function decreasing the forced vital capacity (FVC), such as the increased operative risk and risk of death of these patients following osteoporotic hip fractures $[1,3,14]$.

The prevalence of osteoporosis and osteopenia (low bone mass) in COPD patients varies between $9-69 \%$ and $27-67 \%$, respectively, depending on the diagnostic methods used, the population studied, and the severity of the underlying respiratory disease [3]. In COPD patients, the prevalence of osteoporosis is assumed to be two- to fivefold higher than that in age-matched subjects without airflow obstruction [3]. In a recently developed screening tool for males at risk for
TABLE 1: Factors independent of bone mineral density that increase fracture risk in men [2].

\section{(A) Primary factors}

(i) Previous fragility fracture after 40 years of age, especially vertebral compression fractures*

(ii) Systemic glucocorticoid therapy ${ }^{+}$of $\geq 3$ months' duration

(iii) Advancing age, especially past $65 \mathrm{yr}$

(B) Other key factors

(i) Presence of disease or a condition associated with bone loss

(ii) Family history of osteoporotic fracture

(iii) High alcohol intake: $>2$ units (i.e., $>18$ g) of alcohol daily

(iv) Hypogonadism, primary or secondary

(v) Low BMI $\left(<20 \mathrm{~kg} / \mathrm{m}^{2}\right)$ associated with bone loss

(vi) Smoking, current or past

(vii) Use of LHRH analogs (antiandrogen therapy)

LHRH: luteinizing hormone-releasing hormone, BMI: body mass index.

${ }^{*}$ Height loss of $\geq 6 \mathrm{~cm}$ or kyphosis may be a clinical sign of a vertebral compression fracture;

${ }^{+}$The equivalent of $\geq 7.5 \mathrm{mg} / \mathrm{d}$ of prednisone.

osteoporosis, the presence of COPD is one of the parameters increasing this risk almost four times [15].

\section{Diagnosis of Male Osteoporosis and the Role of Bone Densitometry}

The initial evaluation of COPD men for osteoporosis must include a detailed medical history and physical examination to identify important clinical risk factors that may contribute to bone loss or increase fracture risk in men (Table 1) and to exclude other, except COPD, secondary causes of bone loss in men (Table 2) $[2,6]$. Assessment of COPD severity is necessary based on the patient's level of symptoms, the severity of the spirometric abnormality (Table 3 ), and the presence of complications such as respiratory failure, right heart failure, weight loss, and arterial hypoxemia $[4,12]$. Assessment of serum 25-hydroxy vitamin D (25-OHD), such as other additional tests, as suggested by results of clinical evaluation, should be performed (Table 4) $[2,6]$. Men should have their height measured at baseline and at serial assessments, because height loss may reflect underlying vertebral compression fractures that are often clinically silent $[1,2,6]$. Patients with back pain, height loss, or kyphosis should undergo lateral spinal radiography to detect the presence of vertebral fractures $[2,6]$. The presence of vertebral deformities, clinical vertebral fractures, and other types of bone fracture (pelvis, humerus, ribs, etc.) not associated with important traumas is always suspicious and should lead to a more specific study of the bone status.

Among the intrinsic skeletal factors, low BMD has been consistently associated with an increase in bone fractures. BMD measurement with dual energy X-ray absorptiometry (DEXA) is the accepted reference standard for diagnosis of osteoporosis in men with COPD $[1,2,16,17]$. Considering that COPD is a secondary cause of osteoporosis, it is suggested that COPD men with 3 minor (BMI $<21 \mathrm{~kg} / \mathrm{m}^{2}$, 
TABLE 2: Secondary causes of bone loss in men $[2,6]$.

\begin{tabular}{|c|c|}
\hline Common & Less Common \\
\hline \multirow{2}{*}{$\begin{array}{l}\text { Cushing's syndrome or corticosteroid therapy } \\
\text { (e.g., }>5 \mathrm{mg} \text { /day for }>3 \text { months) }\end{array}$} & Low BMI $\left(<20 \mathrm{Kg} / \mathrm{m}^{2}\right)$ and eating disorders associated with decreased BMI \\
\hline & Lack of exercise or excessive exercise \\
\hline Excessive alcohol use & \multirow{2}{*}{ Antiepileptic drugs (phenytoin, phenobarbitone, primidone, carbamazepine) } \\
\hline Primary or secondary hypogonadism & \\
\hline Low calcium intake and vitamin D deficiency & Thyrotoxicosis or thyroxine overreplacement \\
\hline Smoking & Primary hyperparathyroidism \\
\hline \multirow{2}{*}{ Family history of minimal trauma fracture } & Chronic liver or kidney disease \\
\hline & Malabsorption, including celiac disease \\
\hline \multirow{8}{*}{ Chronic lung disease } & Hypercalciuria \\
\hline & Rheumatoid arthritis or ankylosing spondylitis \\
\hline & Type 1 or type 2 diabetes mellitus \\
\hline & Multiple myeloma or other monoclonal gammopathies \\
\hline & HIV or its treatment with protease inhibitors \\
\hline & Mastocytosis \\
\hline & Organ transplantation or immunosuppressive agents (cyclosporine and tacrolimus) \\
\hline & Osteogenesis imperfecta \\
\hline
\end{tabular}

BMI: body mass index (defined as the weight in kilograms divided by the square of the height in meters).

TABLE 3: Spirometric classification of chronic obstructive pulmonary disease severity based on postbronchodilator $\mathrm{FEV}_{1}[4,11]$.

\begin{tabular}{ll}
\hline Stage I: mild & $\mathrm{FEV}_{1} / \mathrm{FVC}<0.70 \mathrm{FEV}_{1} \geqslant 80 \%$ predicted \\
Stage II: moderate & $\mathrm{FEV}_{1} / \mathrm{FVC}<0.7050 \% \leqslant \mathrm{FEV}_{1}<80 \%$ predicted \\
Stage III: severe & $\mathrm{FEV}_{1} / \mathrm{FVC}<0.7030 \% \leqslant \mathrm{FEV}_{1}<50 \%$ predicted \\
Stage IV: very severe & $\mathrm{FEV}_{1} / \mathrm{FVC}<0.70 \mathrm{FEV}_{1}<30 \%$ predicted or $\mathrm{FEV}_{1}<50 \%$ predicted plus chronic respiratory failure* \\
\hline
\end{tabular}

$\mathrm{FEV}_{1}$ : forced expiratory volume in 1 second; FVC: forced vital capacity

* Respiratory failure: arterial partial pressure of oxygen $\left(\mathrm{Pao}_{2}\right)<8.0 \mathrm{kPa}(60 \mathrm{~mm} \mathrm{Hg})$ with or without arterial partial pressure of $\mathrm{CO}_{2}\left(\mathrm{Paco}_{2}\right)>6.7 \mathrm{kPa}(50 \mathrm{~mm}$ $\mathrm{Hg}$ ) while breathing air at sea level.

current smoking, use of ethanol $>3$ units/day, age $>65$ years, parent hip fracture, rib fracture, inactivity, $\mathrm{FEV}_{1}<50 \%$ predicted) or 1 major [systemic corticosteroids (3 months/year), major fragility fracture (spine-hip)] criteria are at increased risk for osteoporosis and therefore candidates for DEXA [10]. The bone densitometry definition of osteoporosis in men is not as well-standardised as it is in postmenopausal women [18]. However, as in women, BMD measurements, given as area density, but also as T-scores [standard deviations (SD) compared with young adult sex-matched controls] and Zscores (compared with age-and sex-matched controls), are useful for predicting osteoporotic fractures in men $[19,20]$. For every standard deviation (SD) reduction in BMD at the spine or hip, men have a relative risk of fracture that is similar to, or even greater than, the relative risk in women. In contrast, the age-specific prevalence of osteoporosis and fracture is lower in men than in women, and therefore, men have a lower absolute risk for fracture than women at any bone density T-score [20]. Based upon the similar relationship between BMD and fracture in men and women, the World Health Organization (WHO) recommends using the same classification of BMD to define osteoporosis in men (age 50 and older) as in women [21]. The WHO has defined osteopenia (low bone mass) as BMD T-score between -1.0 and $-2.5 \mathrm{SD}$ and osteoporosis as T-score $\leq-2.5 \mathrm{SD}$ [22].

In COPD men, DEXA measurements of the lumbar spine and hip are suggested, because fractures at these sites have the greatest impact on patients' health [1, 17]. DEXA measurement at these two levels, together with the investigation of vertebral deformities using a spine X-ray, has been the techniques most used in clinical trials and in all types of studies on osteoporosis. They are also the most important tools to evaluate the efficacy of antiosteoporotic drugs. Measurement of hip BMD also has the highest predictive value for hip fracture. In addition, if pharmacologic therapy is planned, measurement of lumbar spine BMD is useful as it shows less variability and can detect responses to therapy earlier than hip BMD. We make the diagnosis of osteoporosis based on the lowest T-score measured of either the spine or the hip. Interference from osteophytes and vascular calcifications on the spine measurement are 
TABLE 4: Laboratory tests for the assessment of men with osteoporosis $[2,6]$.

(A) Tests to exclude secondary causes of bone loss

(i) Complete blood count

(ii) Serum calcium

(iii) Albumin

(iv) Liver transaminases

(v) Serum creatinine and calculated creatinine clearance

(vi) Alkaline phosphatise

(vii) Thyroid-stimulating hormone (TSH)

(viii) Testosterone-total, as well as free or bioavailable

(B) Additional tests, as suggested by results of clinical evaluation

(i) Parathyroid hormone (PTH)

(ii) Serum 25-hydroxy vitamin D (25-OHD)

(iii) Serum immunoelectrophoresis

(iv) Celiac antibody testing: gliadin, endomyseal, tissue transglutaminase

(v) 24-hour urine: calcium

(vi) 24-hour urine: free cortisol

common in aging men (after the age of 60 to 65 years) and interfere with the assessment of BMD at this site (artificially high values of BMD in the anterior-posterior directed measurements). In this setting, measurement of hip BMD alone is sufficient. Most clinicians, however, have to rely on one BMD measurement estimating the patients fracture risk.

BMD measurement with DEXA is not universally available, is not portable, and is an imperfect predictor of future fractures [16]. Compared to other risk factors, BMD is at least as good in prediction of fracture as blood pressure measurements to predict stroke and level of cholesterol to predict cardiac infarction [23]. In addition, screening with DEXA may not be cost-effective in all groups [16]. However, available evidence indicates that neither alternative to DEXA (non-DEXA screening tests) is sufficiently sensitive or specific at predicting DEXA-determined bone mass to be recommended as a substitute for DEXA [16].

\section{Fracture Risk Estimation}

BMD assessment, although specific, may not be sensitive for predicting fracture risk, and therefore men with reduced BMD may not necessarily be at an increased risk of fracture. Adding clinical risk factors that are able to predict fracture independent of BMD may improve our ability to predict fracture risk. So, many osteoporosis risk-assessment tools have been developed using risk factors with higher predictive value [1]. For example, the Garvan fracture risk calculator and the WHO fracture risk assessment tool (FRAX) are useful supplements to BMD assessments, as they help physicians to decide which patients might require prolonged treatment to reduce the risk of future fractures $[24,25]$. FRAX is an algorithm that calculates absolute fracture risk based on population-based cohorts from Europe, North America, Asia, and Australia. It is introduced by a WHO task force in 2008 and estimates (calculates) the 10-year absolute fracture risk in individual patients for hip fractures or major osteoporotic fractures (hip, wrist, humerus, and clinical spine) using femoral neck BMD $\left(\mathrm{g} / \mathrm{cm}^{2}\right)$ and easily obtainable clinical risk factors for fracture [25]. The algorithm includes the following risk factors: age, prior fracture, parental history of hip fracture, low body weight or BMI, use of OGCS of $5 \mathrm{mg}$ or more for 3 months or more, rheumatoid arthritis (RA), current cigarette smoking, excessive alcohol intake of 3 units or more daily ( 3 medium glasses of wine or 3 half pints of beer), and secondary osteoporosis. For the FRAX tool, click on Calculation Tool (http://www.shef.ac.uk/FRAX/), and select the country. Based on FRAX tool, the patients with low bone mass are separated into those with low risk versus high risk. So, men aged 50 years and older with low bone mass (T-score between -1.0 and -2.5 SD at the femoral neck or spine) and a 10-year probability of a hip fracture $\geq 3 \%$ or a 10 -year probability of a major osteoporosis-related fracture $\geq 20 \%$ based on the USadapted WHO algorithm, are candidates for medical therapy [25]. Although FRAX has utility as a clinical tool, it also has multiple limitations that may affect fracture risk calculations. Important limitations are the following [26]. (1) The BMD input is limited to the femoral neck based on available population data. FRAX does not account for individuals who have low lumbar T-score but with normal femoral neck. (2) Poor definition of secondary osteoporosis. If BMD is entered, secondary osteoporosis does not change the calculated risk. (3) Certain variables were excluded. For example, FRAX does not include measurements that would be difficult to obtain by a primary care practitioner-measurements of physical activity, vitamin D deficiency, bone turnover markers, or loss of bone mass between sequential BMD measurements. (4) FRAX does not take into account doseresponse relationships. For example, FRAX does not make a difference between single versus multiple fractures, different doses and duration of glucocorticoid use, and different doses of units and duration of use of alcohol or smoking in terms of packs per day. It does not take into account characteristics for prior fractures such as number, severity, and type. (5) FRAX may only be used in untreated patients. (6) There may be racial or ethnic differences that influence fracture risk not taken into account by FRAX. (7) Variability in fracture rates. Despite these limitations, FRAX is being used globally by physicians to assess fracture risk.

Due to disruption in collagen structure, bone architecture and osteocyte network, in addition to demineralization by CS treatment, the increased fracture risk in OGCS users seems to be mostly independent of BMD [1]. This explains why adjustments for BMD have not changed substantially the relative fracture rate in OGCS and why CS users compared to nonusers have higher risk for vertebral fractures at the same levels of BMD. However, bone loss due to OGCS can be measured by BMD.

Markers of bone turnover, like C-terminal cross-linked telopeptide of type 1 collagen or bone alkaline phosphatase, are of uncertain clinical relevance and have not been shown 
to predict the risk of fracture or to correlate with changes in BMD $[1,2,27]$. They might, however, reflect changes related to antiresorptive therapy more rapidly than changes in BMD. Although these biochemical markers have contributed to our knowledge of the pathophysiology and treatment of osteoporosis, at this time they are not recommended in the clinical assessment of men with osteoporosis [2].

\section{Prevention and Treatment of Osteoporosis in COPD Patients}

The prevention and treatment of osteoporosis in COPD men should be to avoid further bone loss and fragility fractures $[1,3]$. This involves nonpharmacologic and pharmacologic measures, as lifestyle measures and nutritional recommendations, management of COPD treatment (based on the use of limited CS doses), and drug therapy (antiresorptive and anabolic drugs).

5.1. Lifestyle Measures and Nutritional Recommendations. In general, preventive and lifestyle modifications should be encouraged for all COPD men to avoid bone loss. A weightbearing and strengthening exercise performed at least 3 times per week may be effective for skeletal health given the association of reduced physical activity with bone loss and fracture in older men, such as the positive effect of exercise in women with osteoporosis $[1,6]$. However, a weight-bearing and strengthening exercise cannot be performed in case of severe COPD. In addition, smoking and excessive alcohol intake should be avoided, while rehabilitation programs in moderate to severe COPD, such as counselling on fall prevention, are useful.

As a general rule, everyone COPD man with or without diagnosed osteoporosis should receive calcium $(1000 \mathrm{mg} /$ day $)$ and vitamin D (800 IU/day) as a standard supplementation, considering that calcium and vitamin $\mathrm{D}$ have been shown to reduce fracture risk in men and women (target serum level of $25-\mathrm{OHD} \geq 30 \mathrm{ng} / \mathrm{mL}$ ) $[1,10]$. Interestingly, a dose-dependent association between vitamin D levels and pulmonary function has been found, and vitamin $\mathrm{D}$ supplement might extend beyond the protective effect on bone and influence the inflammation in the lungs and interfere with comorbidities of COPD [28]. It is recommended as a more aggressive repletion regimen when 25-OHD levels are below $10 \mathrm{ng} / \mathrm{mL}$ [10]. However, in most cases, the addition of pharmacologic therapy is necessary for the treatment of osteoporosis in men.

5.2. Management of COPD Treatment. The recommendations for the management of COPD treatment are mainly based on the use of limited CS doses or reduced CS exposure whenever possible and conclude the following [1]:

(a) long-acting bronchodilators to all patients where airway obstruction might limit the level of physical activity,

(b) ICS or ICS/long-acting $\beta 2$ agonist (LABA) or longacting anticholinergics in severe COPD in order to reduce frequency of exacerbations, (c) maintenance therapy: ICS in moderate doses,

(d) exacerbations: consider dependent on the individual patient:

(1) lower OGCS dose (30 $\mathrm{mg}$ prednisolone for 7 to 8 days)

(2) high-dose ICS/LABA after one dose of prednisolone $30 \mathrm{mg}$.

5.3. Pharmacologic Therapy. Pharmacologic therapy is indicated in COPD men with documented fragility hip or vertebral (clinical or morphometric) fracture, or T-score below $-2.5 \mathrm{SD}$ or with less marked bone loss $(-1<\mathrm{T}$ score $<-2.5)$ and 1 major criteria, as above mentioned [10]. The updated National Osteoporosis Foundation (NOF) guide based upon the WHO fracture prediction algorithm (FRAX), in conjunction with an updated US-specific economic analysis, provides also treatment recommendations for men with osteopenia on BMD (T-score between -1.0 and $-2.5 \mathrm{SD}$ ) [6, 29]. In these individuals, the model suggests that treatment is cost-effective when the 10-year probability of hip fracture reaches 3 percent or the 10 -year probability of major osteoporotic fracture is $\geq 20$ percent [29]. Although these guidelines are useful, osteoporosis treatment should remain individualized through shared decision-making between patient and clinician.

An oral bisphosphonate, as alendronate and risedronate, currently considered that first-line treatment for osteoporosis in men should be recommended, with patient education regarding potential side effects $[2,5,6,30-32]$. The main effect of bisphosphonates is the inhibition of bone resorption, but they have also been shown to prevent osteoblast and osteocyte apoptosis and could thereby preserve the integrity of the osteocyte network and the osteoblast lifetime $[1,33]$. A number of other bisphosphonates are also available but are used less often for the treatment of osteoporosis in men. Intravenous bisphosphonates, as zoledronic acid ( $5 \mathrm{mg}$ once yearly), offer an alternative option for men who cannot tolerate oral bisphosphonates or who find the dosing regimen more convenient $[6,32]$.

Considering that increased bone loss starts immediately after the beginning of OGCS treatment in a daily dose relationship and fracture risk in OGCS users seems to be mostly independent of BMD; antiresorptive therapy in COPD patients should be considered early during OGCS treatment. In this regard, numerous guidelines, including those of the Royal College of Physicians and the American College of Rheumatology, recommend antiresorptive treatment in corticosteroid-treated patients ( $>3$ months) with DEXAdefined osteopenia ( $\mathrm{T}$ score between -1 and $-2.5 \mathrm{SD}$ ), even in the absence of existing fragility fractures [10, 34, 35]. Vestergaard et al. recommended fracture prevention independent of BMD status in patients reaching a yearly cumulative dose of about 800 to $900 \mathrm{mg}$ prednisolone [36]. This equals 2 courses of prednisolone or a daily dose of $2.5 \mathrm{mg}$ prednisolone for 1 year. Bisphosphonates have been also reported to be the most effective of evaluated agents for managing OGCS-induced osteoporosis, and this effect is enhanced further with concomitant use of vitamin D and 
TABLE 5: Suggested approach for diagnosis and management of male osteoporosis in COPD patients.

Initial evaluation

(i) Detailed medical history and physical examination

(ii) Documentation of height, weight, and BMI

(iii) Assessment of COPD severity

(iv) Definition of major risk factors associated with low BMD and fractures

(v) Exclusion or treatment of other secondary causes of bone loss in men

(vi) Assessment of serum 25-OHD. Additional laboratories tests, as suggested by results of clinical evaluation

(vii) Confirmation with lateral spinal radiography of possible vertebral fracture in patients with back pain, height loss, or kyphosis

BMD measurement with DEXA

(i) Recommendations:

All COPD men with 3 minor (BMI $<21 \mathrm{~kg} / \mathrm{m}^{2}$, current smoking, use of ethanol $>3$ units/day, age $>65$ years, parent hip fracture, rib fracture, inactivity, $\mathrm{FEV}_{1}<50 \%$ predicted) or 1 major [systemic corticosteroids (3 months/year), major fragility fracture (spine-hip)]

(ii) Sites: hip and lumbar spine

(iii) Diagnostic categories of BMD according to WHO: definition of osteopenia (low bone mass) as BMD T-score between -1.0 and $-2.5 \mathrm{SD}$ and definition of osteoporosis as BMD T-score $\leq-2.5 \mathrm{SD}$

(iv) Measurement interval:

(1) 2 years or more to follow loss or treatment effect

(2) 1 year if use of OGCSs without antiresorptive therapy

Fracture risk estimation

(i) Fracture risk assessment tool (FRAX), is a useful supplement to BMD assessments, as it helps physicians to decide which patients might require prolonged treatment to reduce the risk of future fractures

Prevention and treatment of osteoporosis

(i) Lifestyle measures and nutritional recommendations

(1) Smoking cessation or avoidance.

(2) Reduction of alcohol consumption, if excessive

(3) Weight-bearing and strengthening exercise

(4) Counselling on fall prevention

(5) Rehabilitation programs in moderate to severe COPD

(6) Calcium and vitamin D supplementation (1000 mg and 800 IU, respectively) to everyone COPD man

(ii) Management of COPD treatment based on the use of limited CS doses.

(ii) Drug therapy

(1) Bisphosphonates

(2) Anabolic drugs (teriparatide)

OGCS: oral glucocorticosteroids; CS: corticosteroids; ICS: inhaled corticosteroids; BMI: body mass index; WHO: World Health Organization; $\mathrm{FEV}_{1}$ : forced expiratory volume in 1 second; FVC: forced vital capacity; COPD: chronic obstructive pulmonary disease; BMD: bone mineral density; DEXA: dual energy X-ray absorptiometry. calcium. Both alendronate and risedronate are effective in the prevention and treatment of OGCS-induced osteoporosis in both men and women, with improvements in BMD and reduction in fracture risk $[2,5]$. Men with OGCS-induced osteoporosis treated with risedronate have fewer vertebral fractures than those taking a placebo [2].

Anabolic drugs like the human parathyroid hormone (PTH) analogue teriparatide (PTH 1-34) stimulate bone formation through effects on osteoblasts and osteocytes and may therefore more directly target the main pathophysiological mechanism in OGCS-induced osteoporosis. Teriparatide has been found to be superior to alendronate in OGCSinduced osteoporosis, both regarding change in BMD and morphometric vertebral fractures, but there is no evidence for hip fracture reduction [10,37]. This agent is suitable for men with severe osteoporosis (established osteoporosis; T-scores $\leq-2.5 \mathrm{SD}$ in combination with at least one fragility fracture) who continue to fracture after one year of bisphosphonate therapy, or multiple risk factors for fracture, or failed previous treatment (those who cannot tolerate or do not have an adequate response to bisphosphonates) [32, 38]. Treatment should be reserved for these high-risk patients because of the need for daily injection and high cost; there are no real concerns about osteosarcoma risk $[1,6]$ with the use of teriparatide in men. Based upon several trials, which have reported that PTH plus alendronate (either started concurrently or prior to PTH) resulted in no additional benefit for spine or hip BMD compared with PTH alone, we do not recommend concurrent use of bisphosphonates with teriparatide for the management of male osteoporosis.

Monitoring the response to therapy is important for identifying patients who may require a change in therapy. Treatment failure is said to have taken place when there are further fragility fractures despite adherence to treatment for one year and/or BMD declines below pretreatment baseline. While there are a number of approaches to monitoring therapy, there is no consensus on the optimal approach. To confirm treatment response, one repeat DEXA scan is usually recommended after a minimum of two years of treatment, as it takes this long for response to antiresorptive agents to exceed the least significant change in BMD [25]. Furthermore, the BMD may fall in the first year of treatment only to subsequently gain in the second year a phenomenon known as regression to the mean [27]. The use of BMD has the disadvantages of taking two years before a lack of response will be noted, and also the spine can be affected by degenerative changes, especially in patients over 65 years. So, osteophytes and vascular calcifications on the spine might be a limitation to the use of spine DEXA in aging men. The BMD measurement interval in OGCS patients without antiresorptive treatment is recommended being one year [1].

An alternative to BMD measurement for monitoring therapy is to use bone turnover markers, which have a maximum suppression in the order of $50 \%$ within three months of starting therapy [27]. These markers tend to be very variable and influenced by many factors. Moreover, its use to monitor response to therapy is not well studied in men and is not routinely recommended [27]. 
A suggested approach for diagnosis and management of male osteoporosis in COPD patients is presented in Table 5.

\section{References}

[1] A. Langhammer, S. Forsmo, and U. Syversen, "Long-term therapy in COPD: any evidence of adverse effect on bone?" International Journal of Chronic Obstructive Pulmonary Disease, vol. 4, pp. 365-380, 2009.

[2] A. A. Khan, A. B. Hodsman, A. Papaioannou, D. Kendler, J. P. Brown, and W. P. Olszynski, "Management of osteoporosis in men: an update and case example," Canadian Medical Association Journal, vol. 176, no. 3, pp. 345-348, 2007.

[3] L. Graat-Verboom, E. F. M. Wouters, F. W. J. M. Smeen, B. E. E. M. Van Den Borne, R. Lunde, and M. A. Spruit, "Current status of research on osteoporosis in COPD: a systematic review," European Respiratory Journal, vol. 34, no. 1, pp. 209218, 2009.

[4] Global Initiative for Chronic Obstructive Lung Disease (GOLD), "Global strategy for the diagnosis, management, and prevention of chronic obstructive pulmonary disease," updated 2009, http://www.goldCOPD.com.

[5] J. D. Ringe, H. Faber, P. Farahmand, and A. Dorst, "Efficacy of risedronate in men with primary and secondary osteoporosis: results of a 1-year study," Rheumatology International, vol. 26, no. 5, pp. 427-431, 2006.

[6] P. R. Ebeling, "Osteoporosis in men," The New England Journal of Medicine, vol. 358, no. 14, pp. 1474-1482, 2008.

[7] H. X. Jiang, S. R. Majumdar, D. A. Dick et al., "Development and initial validation of a risk score for predicting in-hospital and 1-year mortality in patients with hip fractures," Journal of Bone and Mineral Research, vol. 20, no. 3, pp. 494-500, 2005.

[8] T. T. Dam, S. Harrison, H. A. Fink, J. Ramsdell, and E. Barrett-Connor, "Bone mineral density and fractures in older men with chronic obstructive pulmonary disease or asthma," Osteoporosis International, vol. 21, no. 8, pp. 1341-1349, 2010.

[9] N. R. Jørgensen and P. Schwarz, "Osteoporosis in chronic obstructive pulmonary disease patients," Current Opinion in Pulmonary Medicine, vol. 14, no. 2, pp. 122-127, 2008.

[10] A. Lehouck, S. Boonen, M. Decramer, and W. Janssens, "COPD, bone metabolism, and osteoporosis," Chest, vol. 139, no. 3, pp. 648-657, 2011.

[11] S. C. Manolagas and R. S. Weinstein, "New developments in the pathogenesis and treatment of steroid-induced osteoporosis," Journal of Bone and Mineral Research, vol. 14, no. 7, pp. 1061-1066, 1999.

[12] K. F. Rabe, S. Hurd, A. Anzueto et al., "Global strategy for the diagnosis, management, and prevention of chronic obstructive pulmonary disease: GOLD executive summary," American Journal of Respiratory and Critical Care Medicine, vol. 176, no. 6, pp. 532-555, 2007.

[13] C. A. M. Kulak, V. C. Borba, V. Jorgetti et al., "Skeletal microstructural abnormalities in postmenopausal women with chronic obstructive pulmonary disease," Journal of Bone and Mineral Research, vol. 25, no. 9, pp. 1931-1940, 2010.

[14] C. de Luise, M. Brimacombe, L. Pedersen, and H. T. Sørensen, "Chronic obstructive pulmonary disease and mortality following hip fracture: a population-based cohort study," European Journal of Epidemiology, vol. 23, no. 2, pp. 115-122, 2008.

[15] A. J. Shepherd, A. R. Cass, C. A. Carlson, and L. Ray, "Development and internal validation of the male osteoporosis risk estimation score," Annals of Family Medicine, vol. 5, no. 6, pp. 540-546, 2007.
[16] A. Qaseem, V. Snow, P. Shekelle, R. M. Hopkins Jr., A. Forciea, and D. K. Owens, "Screening for osteoporosis, screening for osteoporosis in men: a clinical practice guideline from the American College of Physicians," Annals of Internal Medicine, vol. 148, no. 9, pp. 680-684, 2008.

[17] L. Graat-Verboom, M. A. Spruit, B. E. E. M. van denBorne, F. W. J. M. Smeenk, and E. F. M. Wouters, "Whole-body versus local DXA-scan for the diagnosis of osteoporosis in COPD patients," Journal of Osteoporosis, vol. 2010, Article ID 640878, 2010.

[18] L. Gennari and J. P. Bilezikian, "Osteoporosis in men," Endocrinology and Metabolism Clinics of North America, vol. 36, no. 2, pp. 399-419, 2007.

[19] P. Gardsell, O. Johnell, and B. E. Nilsson, "The predictive value of forearm bone mineral content measurements in men," Bone, vol. 11, no. 4, pp. 229-232, 1990.

[20] S. R. Cummings, P. M. Cawthon, K. E. Ensrud, J. A. Cauley, H. A. Fink, and E. S. Orwoll, "BMD and risk of hip and nonvertebral fractures in older men: a prospective study and comparison with older women," Journal of Bone and Mineral Research, vol. 21, no. 10, pp. 1550-1556, 2006.

[21] J. A. Kanis, E. V. McCloskey, H. Johansson, A. Oden, L. J. Melton III, and N. Khaltaev, "A reference standard for the description of osteoporosis," Bone, vol. 42, no. 3, pp. 467-475, 2008.

[22] "Assessment of fracture risk and its application to screening for postmenopausal osteoporosis," Report of a WHO Study Group, 1994, World Health Organization Technical Report Series.

[23] J. A. Kanis, "Osteoporosis III: diagnosis of osteoporosis and assessment of fracture risk," Lancet, vol. 359, no. 9321, pp. 1929-1936, 2002.

[24] J. P. W. Van Den Bergh, T. A. C. M. Van Geel, W. F. Lems, and P. P. Geusens, "Assessment of individual fracture risk: FRAX and beyond," Current Osteoporosis Reports, vol. 8, no. 3, pp. 131-137, 2010.

[25] J. A. Kanis, O. Johnell, A. Oden, H. Johansson, and E. McCloskey, "FRAX and the assessment of fracture probability in men and women from the UK," Osteoporosis International, vol. 19, no. 4, pp. 385-397, 2008.

[26] S. L. Silverman and A. D. Calderon, "The utility and limitations of FRAX: a US perspective," Current Osteoporosis Reports, vol. 8, no. 4, pp. 192-197, 2010.

[27] S. P. Tuck and H. K. Datta, "Osteoporosis in the aging male: treatment options," Clinical Interventions in Aging, vol. 2, no. 4, pp. 521-536, 2007.

[28] W. Janssens, A. Lehouck, C. Carremans, R. Bouillon, C. Mathieu, and M. Decramer, "Vitamin D beyond bones in chronic obstructive pulmonary disease: time to act," American Journal of Respiratory and Critical Care Medicine, vol. 179, no. 8, pp. 630-636, 2009.

[29] National Osteoporosis Foundation, Clinician's Guide to Prevention and Treatment of Osteoporosis, National Osteoporosis Foundation, Washington, DC, USA, 2008.

[30] R. Bobba and J. D. Adachi, "Review of the safety and efficacy of risedronate for the treatment of male osteoporosis," Clinical Interventions in Aging, vol. 2, no. 3, pp. 275-282, 2007.

[31] J. D. Ringe, P. Farahmand, H. Faber, and A. Dorst, "Sustained efficacy of risedronate in men with primary and secondary osteoporosis: results of a 2-year study," Rheumatology International, vol. 29, no. 3, pp. 311-315, 2009.

[32] J. D. Ringe, “Osteoporosis in men," Medicographia, vol. 32, no. 1, pp. 71-78, 2010. 
[33] L. I. Plotkin, J. I. Aguirre, S. Kousteni, S. C. Manolagas, and T. Bellido, "Bisphosphonates and estrogens inhibit osteocyte apoptosis via distinct molecular mechanisms downstream of extracellular signal-regulated kinase activation," Journal of Biological Chemistry, vol. 280, no. 8, pp. 7317-7325, 2005.

[34] J. P. Devogelaer, S. Goemaere, S. Boonen et al., "Evidencebased guidelines for the prevention and treatment of glucocorticoid-induced osteoporosis: a consensus document of the Belgian Bone Club," Osteoporosis International, vol. 17, no. 1, pp. 8-19, 2006.

[35] J. Compston, "US and UK guidelines for glucocorticoidinduced osteoporosis: similarities and differences," Current Rheumatology Reports, vol. 6, no. 1, pp. 66-69, 2004.

[36] P. Vestergaard, L. Rejnmark, and L. Mosekilde, "Fracture risk associated with different types of oral corticosteroids and effect of termination of corticosteroids on the risk of fractures," Calcified Tissue International, vol. 82, no. 4, pp. 249-257, 2008.

[37] K. G. Saag, E. Shane, S. Boonen et al., "Teriparatide or alendronate in glucocorticoid-induced osteoporosis," The New England Journal of Medicine, vol. 357, no. 20, pp. 2028-2039, 2007.

[38] J. M. Kaufman, E. Orwoll, S. Goemaere et al., "Teriparatide effects on vertebral fractures and bone mineral density in men with osteoporosis: treatment and discontinuation of therapy," Osteoporosis International, vol. 16, no. 5, pp. 510-516, 2005. 


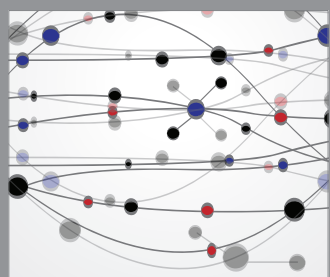

The Scientific World Journal
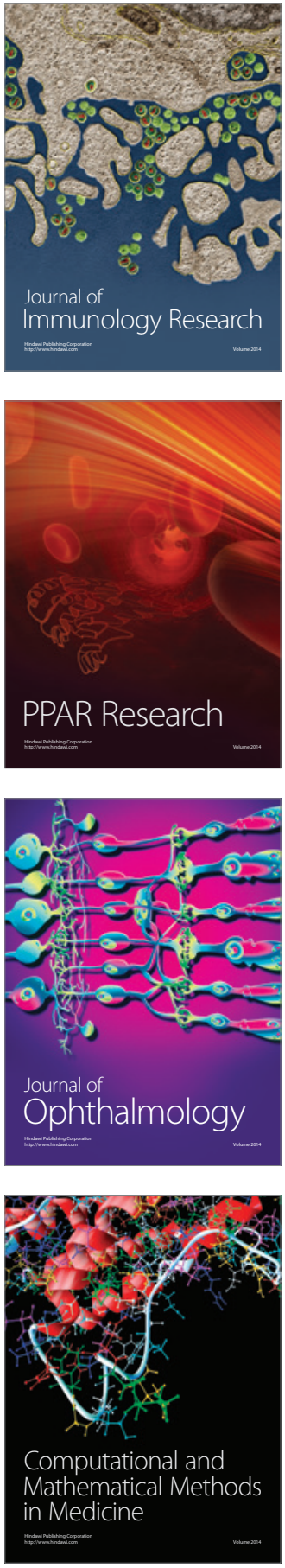

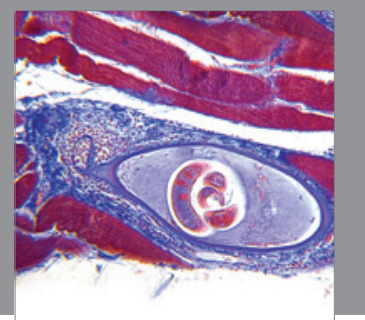

Gastroenterology

Research and Practice
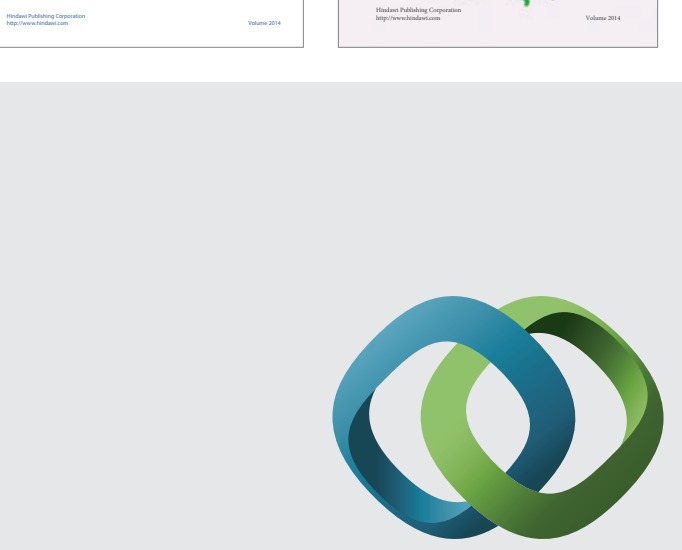

\section{Hindawi}

Submit your manuscripts at

http://www.hindawi.com
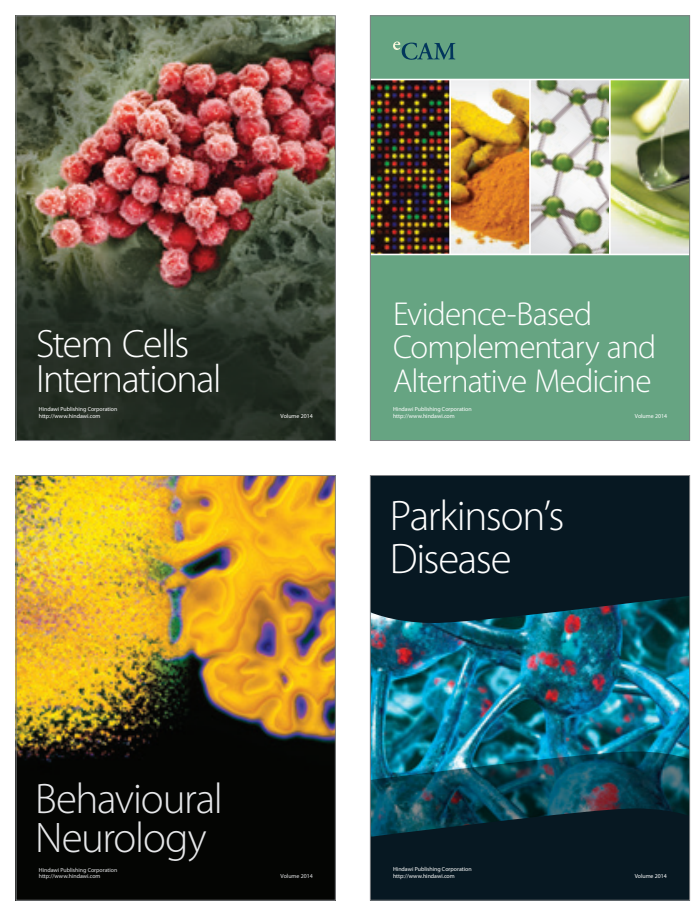

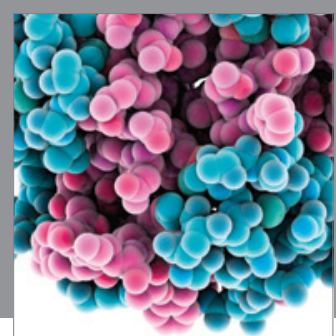

Journal of
Diabetes Research

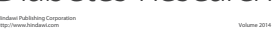

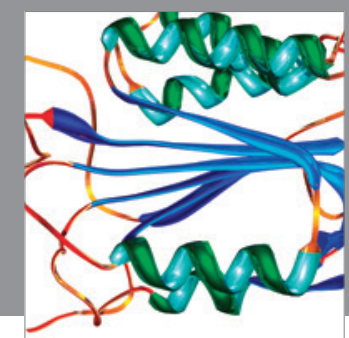

Disease Markers
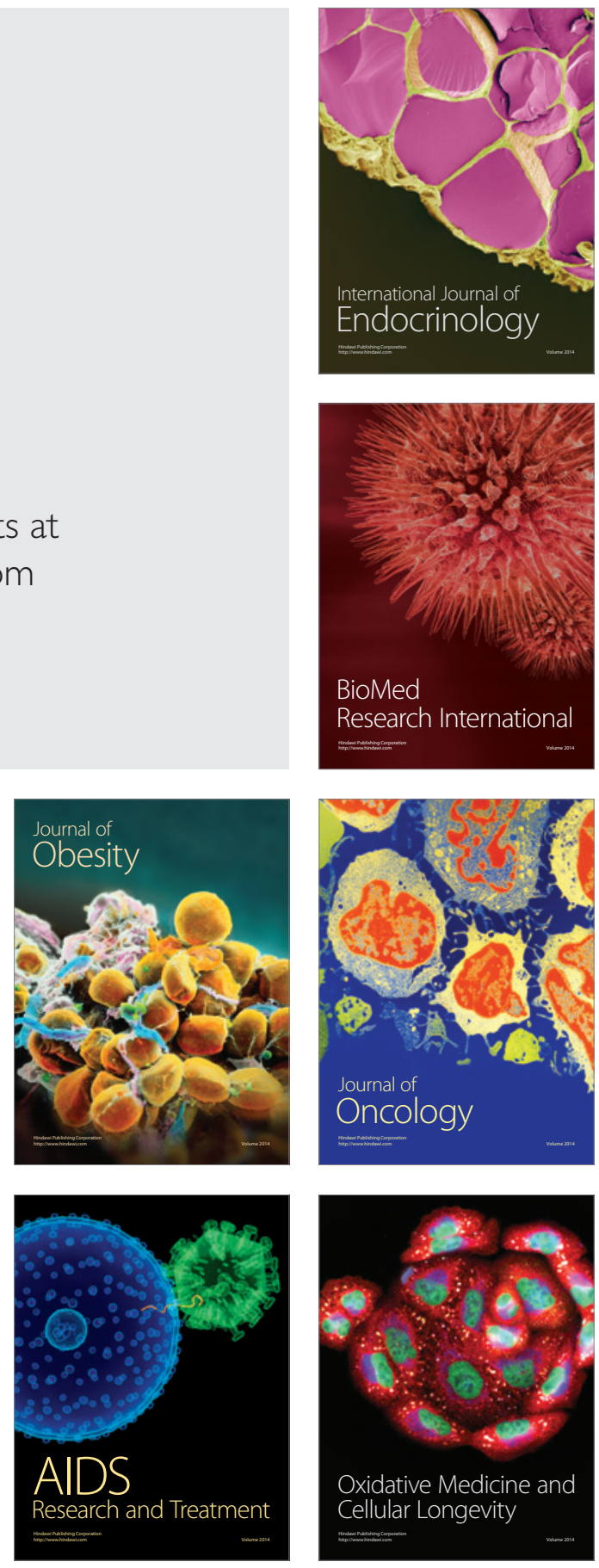